\title{
CRESCIMENTO EM ALTURA DO PAU-FERRO (Astronium balainae) EM REFLORESTAMENTO
}

\author{
HEIGHT GROWTH OF PAU-FERRO (Astroniumn balansae) \\ IN A PLANTATION FOREST
}

\author{
César Augusto Guimarães Finger ${ }^{1}$ Rute Berger ${ }^{2}$ \\ Jackson Roberto Eleotério ${ }^{2}$ Paulo Renato Schneider ${ }^{3}$
}

\section{RESUMO}

Foi avaliado, através da técnica de análise de tronco, o crescimento em altura do pau-ferro (Astronium balansae), em reflorestamento com doze anos de idade, no municipio de São Sepé - RS, permitindo quantificar e descrever o crescimento cumulativo e em forma de taxas anuais médias e correntes em função da idade. Os dados de uma árvore do estrato dominante e uma do estrato dominado foram modelados através do procedimento "Stepwise" de regressão, sendo obtidas equações de alta precisão e ajuste para descrever o crescimento, o incremento médio e corrente anual da espécie. No periodo de tempo coberto pelos dados, o culminio do incremento corrente em altura ocorreu aos seis anos e do incremento médio entre o oitavo e nono ano, na árvore dominante. não devendo entretanto, esta constatação ser tomada como definitiva antes da medição do crescimento em árvores mais velhas, o que permitirá verificar ou não a continuidade da queda das taxas de incremento. Não foi possivel determinar as idades de culminio do incrementos na árvore dominada.

Palavras-chave: Astronium balansae, altura, incremento, modelagem.

\section{SUMMARY}

The growth in height of Astronium balansae, in a twelve years old plantation stand, in the county of São Sepé-RS, was evaluated by a trunk analyses, allowing to quantify and describe the cumulative growth and the average annual and the current rates as a function of the tree age. The data from one dominant tree and from one dominated were modeled by a stepwise regression procedure. High precision equations were fitted to describe the height growth of the specie. During the time period observed, culminating of the current increment in height happened at six years and the respective average increment between the eighty and ninety years age. in the dominant tree. However. this observation should not be taken as final; only after determinatios in older trees are made, which will verify or not the decline in the growth rates. It was no possible to determine the ages at which the incremements will culminate in the dominated tree.

Key words: Astronium balansae, heigth, increment, modelling.

\section{INTRODUÇÃO}

$\mathrm{Na}$ busca de alternativas regionais para suprir a demanda de madeira de qualidade com espécies tradicionais, o pau-ferro, Astronium balansae, adquire particular importância. Sua madeira tem uso generalizado em postes, moirões e estruturas afins por apresentar alta resistência natural ao apodrecimento. Apresenta também elevada dureza e alta resistência à flexão e ao choque. Como lenha também é apreciada apresentando poder calorífico superior à $4500 \mathrm{cal} / \mathrm{g}$

\footnotetext{
${ }^{1}$ Engenheiro Florestal, Professor Adjunto, Doutor, Departamento de Ciências Florestais da Universidade Federal de Santa Maria - UFSM. 97105-900 - Santa Maria - RS. E-mail: finger@ccr.ufsm.br. Autor para correspondência.

2 Acadêmicos do Curso de Graduação em Engenharia Florestal, bolsistas do PIBIC-CNPq

${ }^{3}$ Engenheiro Florestal, Professor Titular, Doutor, Departamento de Ciências Florestais da UFSM. Recebido para publicação em 30.04.97. Aprovado em 19.11.97
} 
(REITZ et al.,1988; BELTRÃO et al.,1984; SCHULTZ, 1953 ).

A espécie ocorre naturalmente na região da Campanha do Rio Grande do Sul, preferencialmente ao longo do Vale do Rio Ibicuí, em locais com afloramentos rochosos associados a solos mais evoluídos. Neste habitat pedregoso, formam maciços com até $85 \%$ do volume comercial com casca da floresta (LONGHI,1987). As árvores são decíduas, medianas, comumente de 10 a 15 metros de altura e 20 a 40 centímetros de diâmetro; geralmente apresentam tronco um pouco tortuoso e casca densamente cortiçosa, estriada no corte transversal (REITZ et al., 1988; SCHULTZ, 1953). Seu crescimento é classificado como rápido, com diâmetro médio de $15 \mathrm{~cm}$ em 23 anos (SCHULTZ, 1953).

O crescimento da espécie com relação as variáveis dendrométricas diâmetro, altura e volume é desconhecido não havendo medições periódicas destas variáveis que permitam descrever esta grandeza. Desta forma, este estudo é um subsídio à silvicultura e ao manejo da floresta, pois fornecerá informações preliminares sobre o potencial de crescimento em altura, permitindo quantificar o tempo necessário para que a árvore alcance uma determinada dimensão. Considerando que para conhecer tais características do crescimento de uma espécie é necessário acompanhar com medições periódicas todo seu ciclo de vida, implicando em um longo período de observações, a análise de tronco assume particular importância, pois é uma alternativa rápida, eficaz e precisa, que permite conhecer, pelo exame e medições dos anéis anuais de crescimento tomados em diversas posições ao longo do tronco da árvore, as dimensões alcançadas por esta em épocas passadas. Através da contagem e medição de anéis de crescimento podem ser obtidas informações sobre os parâmetros biométricos importantes para descrever o desenvolvimento da espécie como o diâmetro, altura e volume.

Assim, neste estudo procurou-se quantificar o crescimento de Astronium balansae pelo emprego da técnica da análise de tronco e descrever o crescimento em altura e os incrementos corrente e médio anual para a árvore do estrato dominante e dominado.

\section{MATERIAIS E MÉTODOS}

O estudo do crescimento Astronium balansae foi realizado a partir da coleta de fatias de 2 árvores pertencentes a 2 estratos arbóreos (uma no dominante e uma no dominado) de um povoamento homogêneo da espécie, com 12 anos de idade, implantado no Município de São Sepé -RS.
As árvores foram abatidas e delas retiradas fatias, com espessura de aproximadamente $4 \mathrm{~cm}$, ao longo do tronco nas posições de 0,$16 ; 1,30 ; 2,30 ; 3,40$; 4,$40 ; 5,40 ; 6,50$ e 7,60 metros de altura, na árvore dominante e à 0,$11 ; 1,30 ; 2,30 ; 3,10 ; 3,80$ e 4,80 metros de altura na árvore dominada.

Nas árvores foram também medidas as alturas totais. As fatias foram secas em estufa, plainadas e lixadas para facilitar a visualização dos anéis. A seguir procedeu-se a medição dos anéis sobre quatro raios partindo-se da medula até a casca. O primeiro raio foi traçado coincidindo com o maior diâmetro da fatia e os demais, no sentido horário em relação a este, sempre mantendo perpendicularidade entre eles. Os raios foram medidos com precisão de milímetro e anotados em formulário próprio apresentados por FINGER (1992). Desta forma, cada anel de crescimento foi medido quatro vezes e o valor médio deste forneceu o raio e diâmetro da árvore em cada idade e em cada posição onde foram cortadas as fatias.

Providos dos valores de diâmetro em cada idade em diferentes alturas, pôde-se construir o perfil longitudinal de cada árvore obtendo-se a altura para cada idade, além de permitir visualizar os diâmetros e distribuição dos anéis nas diferentes idades. O perfil da árvore foi desenhado em um eixo coordenado, mantendo-se o eixo " $y$ ", correspondente a altura da árvore, em escala 1:30 e o eixo " $x$ ", dos diâmetros, em escala 1:100.

A partir dos valores de altura foram calculados, para cada árvore, os incrementos correntes anuais (ICA) e incrementos médios anuais (IMA) em altura com o emprego das expressões:

$$
I C A=Y_{m}-Y_{m-1} \quad \text { e } \quad I M A=\frac{Y_{m}}{m}
$$

onde $Y_{m}$ é grandeza da variável no ano considerado e, $Y_{m-1}$ é a grandeza da variável no ano anterior; $m=$ idade.

Na seqüência, os dados altura, ICA e IMA foram empregados como variáveis dependentes em modelos matemáticos, tendo a idade como variável independente. Para tal foi empregado o procedimento "Stepwise" de regressão, utilizando como variáveis dependentes e independentes, tanto variáveis puras, como também, a forma transformada recíproca $\mathrm{e}$ logaritmica.

Ainda, para descrever a relação do incremento sobre a idade das árvores, foi calculada a derivada primeira e segunda da função de crescimento ajustada para estimar, respectivamente, o incremento corrente e médio anual. 
Outra estimativa destas grandezas foi feita com o cálculo dos incrementos médio e corrente a partir das estimativas geradas na curva de crescimento (ajustada). Posteriormente, os valores de IMA e ICA, recalculados, foram empregados no procedimento "Stepwise" de regressão para determinação de novos modelos matemáticos.

Na seleção dos modelos foi considerado o coeficiente de determinação $\left(\mathrm{R}^{2}\right)$, o erro padrão em percentagem da média (Syx\%) e a análise de resíduos.

\section{RESULTADOS E DISCUSSÕES}

Os modelos matemáticos desenvolvidos pelo procedimento "Stepwise" de regressão para descrever o crescimento em altura das árvores de pauferro em função da idade alcançaram grande precisão e ajuste (Tabela 1). O modelo selecionado para descrever o crescimento da árvore pertencente ao estrato dominante foi o de número 1 , pois apresentou o maior coeficiente de determinação $\left(\mathrm{R}^{2}=99,9 \%\right)$ e o menor erro padrão da estimativa em percentagem da média (Syx $=0,9 \%$ ), quando comparado ao modelo logarítmico calculado para mesma árvore (modelo 2 ).

O mesmo resultado foi apresentado para a modelagem dos dados da árvore do estrato dominado, onde o modelo aritmético e recíproco (modelo 3), apresentou maior coeficiente de determinação $\left(R^{2}=99,1 \%\right)$ e o menor erro padrão da estimativa em percentagem da média ( $\mathrm{Syx}=2,6 \%$ ), quando comparado ao modelo logarítmico (modelo 4).

Os testes de resíduos, realizados graficamente, não evidenciaram tendências do modelo de regressão, permitindo ratificar a seleção do modelo.
O ajuste do modelo pode também ser visto na Figura 1 , onde se verifica o excelente ajuste alcançado pelos modelos matemáticos para as árvores dominante e dominada.

As derivadas primeira e segunda dos modelos 1 e 3 em relação a variável idade, selecionados para descrever o crescimento em altura da árvore dominante e dominada, expressam o incremento corrente e médio anual a partir das estimativas da curva de crescimento, porém apresentam-se complexas e de díficil emprego quando comparadas com as funções geradas por modelagem, não sendo, por isso, apresentadas neste trabalho.

Os modelos desenvolvidos por "Stepwise" para descrever o incremento, tendo como variáveis dependentes os valores observados, apresentaram para o ICA coeficientes de determinação relativamente baixos, variando entre 63 e $69 \%$, com erro padrão em percentagem da média altos, entre 27 e $105 \%$.

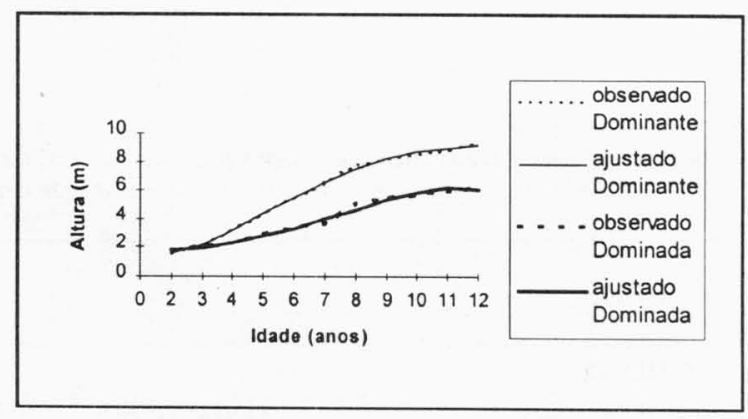

Figura 1 - Valores observados e ajustados de altura em função da idade para a árvore dominante e dominada.

Tabela 1 - Modelos calculados para descrever a altura (h) em função da idade (t), para as árvores dos estratos dominante e dominado.

\begin{tabular}{|c|c|c|c|c|}
\hline Árvore & $\mathrm{n}^{\circ}$ & Modelo & $\mathrm{R}^{2}$ & Syx $\%$ \\
\hline \multirow[t]{2}{*}{ DOMINANTE } & 1 & $h=\left(-0,505889+0,003025 t^{2}+2,100611 t^{-1 / 2}-0,000119 t^{3}-1,478916 \cdot t^{-3}\right)^{-2}$ & 99,9 & 0,9 \\
\hline & 2 & $h=\exp \left(-0,941388-0,000335^{*} t^{3}+2,676046^{*} t^{-3}+1,498327^{\prime} \ln (t)\right)$ & 99,8 & 5,4 \\
\hline \multirow[t]{2}{*}{ DOMINADA } & 3 & $h=\left(1,439059+0,459978^{\circ} \ln (t)-0,714178 \sqrt{t}+0,000174^{\prime} t^{3}\right)^{-2}$ & 99,1 & 2,6 \\
\hline & 4 & $h=\exp \left(0,420994+0,242640^{\circ}[\ln (t)]^{2}\right)$ & 97,1 & 6,3 \\
\hline
\end{tabular}


Segundo os critérios de seleção adotados, o melhor modelo para descrever o ICA da árvore dominante é o de número um, que apresentou $\mathrm{R}^{2}$ igual a $64,7 \%$ e Syx de $27,7 \%$. Para a árvore dominada, observa-se na Tabela 2, que as estatísticas de $\mathrm{R}^{2} \mathrm{e}$ Syx $\%$ apresentam valores iguais, respectivamente, $68,9 \%$ e $51,8 \%$. Entretanto foi selecionado o modelo de número 4, da Tabela 2, para descrever a relação do ICA e a idade, devido a sua maior simplicidade de aplicação.

Os modelos selecionados para a árvore dominante e dominada tiveram os resíduos avaliados de forma gráfica, não tendo sido encontradas tendências de estimativas. Os valores percentuais altos mostrados pelo Syx são devidos a grande variação anual dos incrementos e não a tendências do modelo.

A modelagem para descrever o incremento médio anual em altura para a árvore dominante, apresentada na Tabela 2 , mostrou o modelo de número 5 como o de menor $\operatorname{Syx}(5,7 \%)$ e de maior $\mathrm{R}^{2}$ $(96,4 \%)$. Para os dados da árvore dominada, foi selecionado o modelo número 7 , o qual apresentou $\mathrm{R}^{2}$ de $86,9 \%$ e Syx de $6,6 \%$. A aplicação de testes gráficos de resíduos não evidenciaram qualquer tendência que pudesse descaracterizar os modelos para descrever o incremento.

A modelagem contendo como variável dependente os valores de IMA e ICA calculados a partir das funções apresentadas na Tabela 1 e 2 permitiu, como esperado pela eliminação do efeito do crescimento anual, obter funções com maior precisão e ajuste para descrever o incremento corrente e médio anual conforme apresentado na Tabela 3 .

A análise das Figuras 2 e 3 , construídas com os dados observados e ajustados pelos modelos de regressão, permitem compreender a magnitude do valor de Syx do modelo selecionado para descrever o ICA da árvore dominante e dominada (modelos 1 e 4 da Tabela 2). Verifica-se no gráfico a grande queda no valor da taxa de incremento corrrente entre o oitavo e o décimo primeiro ano, período em que o modelo superestimou as estimativas da árvore dominante. $\mathrm{Na}$ árvore dominada, também a grande variação da taxa de crescimento anual no oitavo ano, explica o valor alto encontrado para o Syx. Por outro lado, o ajuste do modelo de incremento médio foi muito bom para a árvore dominante e para a dominada.

Tabela 2 - Modelos matemáticos calculados para descrever o incremento corrente anual (ICA) e médio anual (IMA) em altura em função da idade(t). para as árvores dos estratos dominante e dominado.

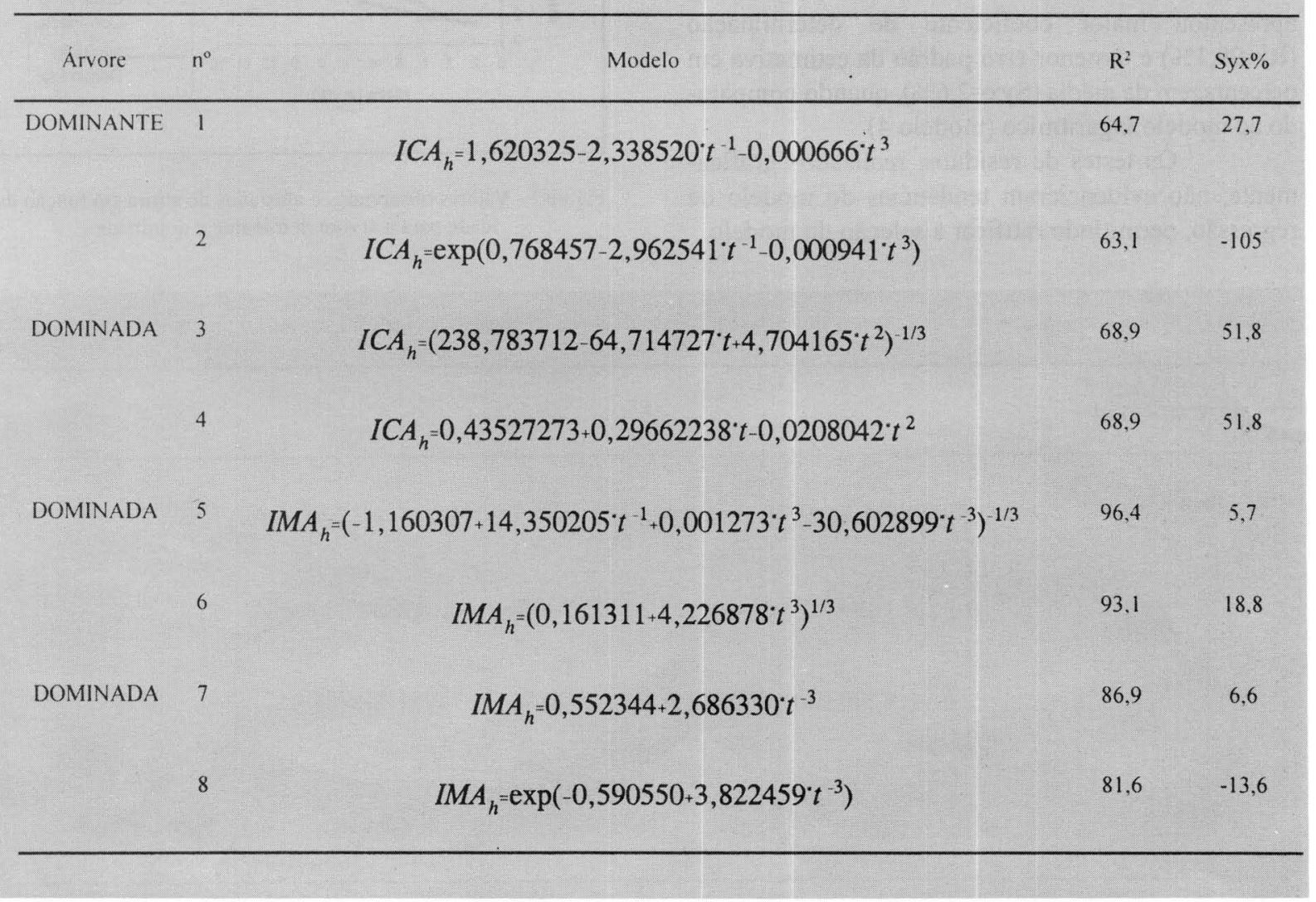


Tabela 3 - Modelos matemáticos calculados para descrever o incremento médio anual (IMA) e corrente anual (ICA) em altura, para árvores dos estratos dominante e dominado, a partir do ICA e IMA obtidos na função de crescimento.

\begin{tabular}{|c|c|c|c|c|c|}
\hline Árvore & $n^{0}$ & Modelo & & $\mathrm{R}^{2}$ & Syx\% \\
\hline DOMINANTE & 1 & $I M A_{h}=1,6428-4,5519^{\prime} t^{-1}-0,0038^{\prime} t^{2}+5,6772^{\prime} t^{-2}$ & & 98,9 & 0,00009 \\
\hline & 2 & $I C A_{h}=\exp \left(0,6525-0,0012 \cdot t^{3}-9,4294^{*} t^{-2}\right)$ & & 99,4 & $-0,00900$ \\
\hline \multirow[t]{2}{*}{ DOMINADA } & 3 & $I C A_{h}=-32,1344+3,4361^{\prime} t+151,6411^{\prime} t^{-1}-0,1366^{\circ} t^{2}-351,2821^{\prime} t$ & $+316,7073 t^{-3}$ & 99,9 & 0,00683 \\
\hline & 4 & $I M A_{h}=13,8116-3,2937 * t^{-2}+3,2746 * \ln (t)^{2}-9,6659 *$ & $* \sqrt{t}$ & 99,7 & 0,00818 \\
\hline
\end{tabular}

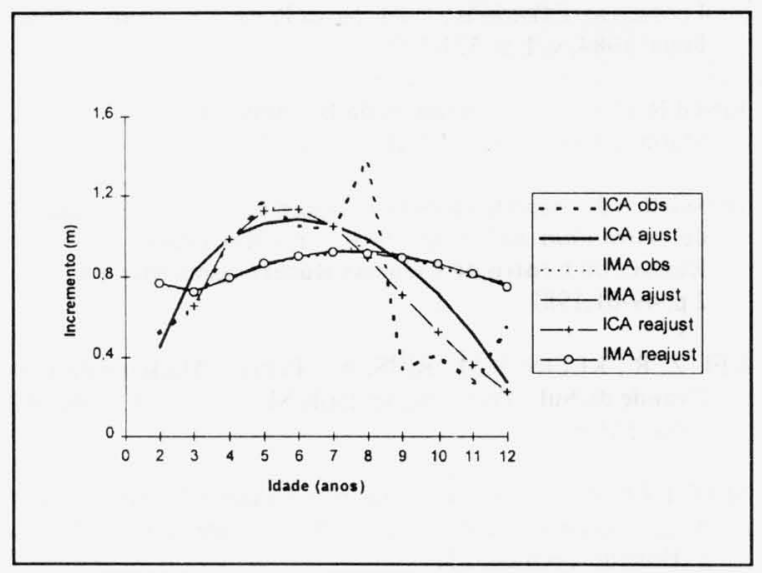

Figura 2 - Incrementos corrente (ICA) e médio anual (IMA) em altura observados e ajustados em função da idade para a árvore dominante.

As curvas geradas pelos modelos apresentados na Tabela 3, modelos estes reajustados a partir das estimativas de ICA e IMA da função de crescimento, mostraram curvas de bom ajuste quando comparadas com os dados observados, tanto para a árvore dominante como para a dominada. Nas Figuras 2 e 3 pode ser observado que a curvas de IMA são quase totalmente coincidentes para qualquer dos métodos de estimativa. Já para as curvas de ICA (Figura 2) verifica-se que as estimativas obtidas pelas duas funções ajustadas diferem entre si, mas são igualmente consistentes quando comparadas com os valores observados.
As curvas de ICA ajustadas para a árvoredominada mostram, entretanto, grande diferença entre si (Figura 3). Os valores gerados pela função ajustada aos dados de incremento calculados a partir da curva de crescimento mostram melhor ajuste em relação aos dados observados até a idade de 11 anos. A partir de então os valores de ICA calculados aproximam-se de zero, subestimando os incrementos observados.

A utilização de funções de ICA e IMA reajustadas a partir de dados da curva de crescimento traz a vantagem de permitir obter o mesmo valor numérico de incremento através da função e através

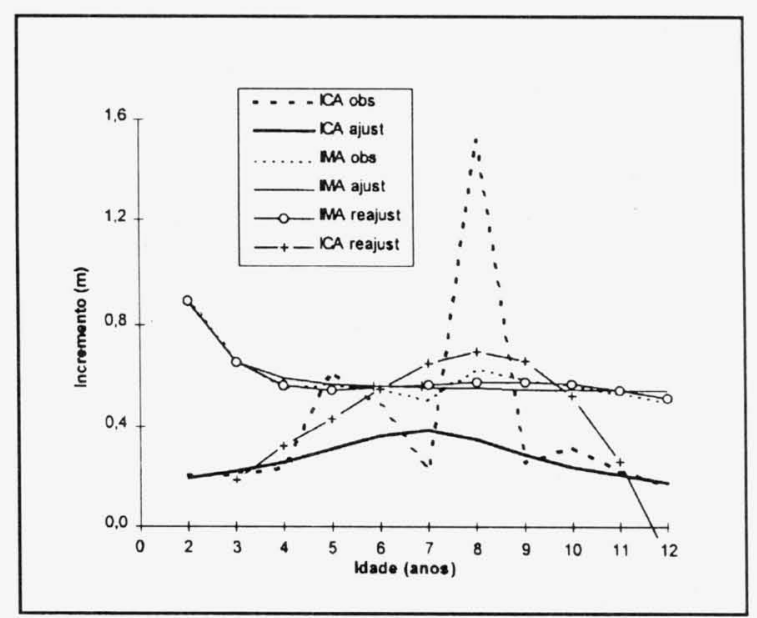

Figura 3 - Incrementos corrente (ICA) e médio anual (IMA) em altura observados e ajustados em função da idade para a árvore dominada 
da curva de crescimento, ou seja, pelo acúmulo dos valores das funções reajustadas se obtém novamente o valor da curva de crescimento, o que muitas vezes não é obtido quando se estima com funções baseadas nos valores observados de IMA e ICA.

De acordo com os modelos ajustados e considerando o período de observação dos dados, 12 anos, a árvore dominante atinge o máximo ICA aos seis anos e o máximo IMA entre o oitavo e nono ano. Entretanto, esta constatação só deve ser tomada como definitiva após a medição do crescimento em árvores mais velhas, próximo ao período de rotação, o que permitirá verificar ou não a continuidade da queda das taxas de incremento na árvore dominante.

Não foi possível identificar, com base nos dados observados, os pontos de culmínio destes incrementos na árvore dominada devido a pouca variação nas taxas de incremento desta árvore ao longo do periodo de observação.

As menores taxas de crescimento anual e a relativa constância ao longo da variação da idade encontadas na árvore dominada é característica de árvores estressadas, que compõem o último estrato arbóreo e que sofrem sofrem menor influência com a variação das condições locais do sítio ano a ano.

\section{CONCLUSÕES}

A quantificação do crescimento de Astronium balansae, com o emprego da técnica de análise de tronco e aplicação de método de modelagem pelo procedimento "Stepwise" de regressão, permite descrever o crescimento e os incrementos médio e corrente anual da espécie com grande preci- são; a derivação das funções de crescimento resultam em modelos de maior complexidade e de difícil emprego para a estimativa do incremento; a determinação de funções de incremento, a partir de taxas anuais calculadas da função cumulativa permite aumentar a precisão e ajuste dos modelos; o culmínio do incremento corrente em altura ocorre aos seis anos e do incremento médio entre o oitavo e nono ano, na árvore dominante; não é possível determinar, na árvore dominada, as idades de culmínio do incrementos; e, os resultados e modelos matemáticos desenvolvidos representam, com alta precisão, a realidade das árvores amostradas, sendo necessário, porém, o aumento do número de árvores amostras para a melhor representação da espécie na região.

\section{REFERÊNCIAS BIBLIOGRÁFICAS}

BELTRÃO, L., VASCONCELlOS, J.M. de, FROSI, R. Desenvolvimento de tecnologia para exploraçăo florestal do "Pauferro" (Astronium balansae Engl.). In.: $5^{\circ}$ CONGRESSO Florestal Estadual, 1984. Nova Prata, RS. Anais ...Nova Prata, 1984, v. I, p. 521-523.

FINGER, C.A.G. Fundamentos de Biometria Florestal. Santa Maria: UFSM/CEPEF/FATEC, 1992. 269 p.

LONGHI, S.J. Aspectos fitossociológicos de uma floresta natural de Astronium balansae ENGL., no Rio Grande do Sul. Revista do Centro de Ciências Rurais, Santa Maria, v. 7 n. 2 p. 49-61,1987.

REITZ, R., KLEIN, R.M., REIS, A. Projeto Madeira do Rio Grande do Sul. Porto Alegre: HBR/SUDESUL/SAA-DRNR, 1988. $525 \mathrm{p}$.

SCHULTZ, A.R. Pau-ferro no Rio Grande do Sul. Porto Alegre: Instituto Tecnológico do Rio Grande do Sul, 1953.9 p (Boletim Técnico, 23).

Ciência Rural, v. 28, n. 2, 1998. 